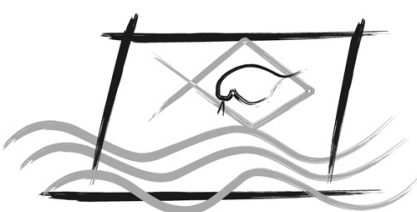

ECOTOX - BRASIL

\title{
Disposição continental de resíduos de mineração de carvão: drenagem ácida, ecotoxicidade aguda e biodisponibilidade de metais
}

\author{
R.G. Cesar ${ }^{1,2}$; M.B. Coelho'; T.T. Alvaro 3 ; J.P. Colonese ${ }^{2}$; \\ Z.C. Castilhos ${ }^{2}$; S.G. Egler ${ }^{2}$; E.D. Bidone ${ }^{1}$; H. Polivanov ${ }^{3}$ \& N.Z Alexandre ${ }^{4}$ \\ ${ }^{1}$ UFF. Instituto de Química, Departamento de Geoquímica Ambiental.Niterói, Rio de Janeiro - RJ. \\ ${ }^{2}$ CETEM/MCT. Serviço de Desenvolvimento Sustentável. Rio de Janeiro - RJ. \\ ${ }^{3}$ UFRJ. CCMN-Instituto de Geociências. Departamento de Geologia. Rio de Janeiro - RJ. \\ ${ }^{4}$ IPAT - Universidade do Extremo Sul Catarinense. Criciúma - SC.
}

(Received May 24, 2012; Accept April 22, 2013)

\begin{abstract}
Terrestrial disposal of coal mining residues: acid drainage, acute ecotoxicity and bioavailability of heavy metals - Potential bioavailability and acute ecotoxicity associated with terrestrial disposal of coal mining residues were studied through acute bioassays with earthworms (Eisenia andrei), lettuce (Lactuca sativa) and aquatic micro-crustaceans (Daphnia similis). A composed sample of coal mining residue was collected at some surface deposits at the Urussanga river Basin, one of the three basis of the Southern Santa Catarina Coal Basin, an area highly degraded by mining activities. The bioassays were performed according to procedures described in standard protocols (ASTM, ISO and ABNT). In a hypothetic risk scenario of pyrite acute exposure, the results indicated low levels of ecotoxicity and low bioavailability of toxic metals for earthworms. Significant effects on the germination and morphology of $L$. sativa were only detected for high rates $(\sim 50 \%)$ of residue application on the soil. It is probable that such low toxicity levels are related to the short time of pyrite exposure to oxygen, generating a less intense acid drainage. For D. similis, significant damages (immobility) were observed for doses higher than $25 \%$ in elutriates, suggesting the occurrence of adverse effects in scenarios where such coal mining residues could be leached.
\end{abstract}

Keywords: Earthworms, Lettuce, Micro-crustaceans, Bioassays, Acidic Mining Drainage (AMD).

\section{INTRODUÇÃO}

O carvão mineral explotado da Formação Rio Bonito (idade permiana, Bacia do Paraná) representa menos de $2 \%$ da fonte para geração de energia elétrica no Brasil (Castilhos et al. 2010). Entretanto, a exposição dos minerais de pirita $\left(\mathrm{Fe}_{2} \mathrm{~S}\right)$ (presentes nos rejeitos e até mesmo no estéril do carvão e dispostos sobre os solos) ao oxigênio e água permite a formação de ácido sulfúrico $\left(\mathrm{H}_{2} \mathrm{SO}_{4}\right)$, desencadeando o fenômeno da drenagem ácida de minas (DAM). A DAM é um dos principais impactos ambientais da mineração de carvão e se caracteriza pela lixivição de metais (e outros agentes tóxicos) existentes no minério, decrescendo dramaticamente o $\mathrm{pH}$ e contaminando ecossistemas terrestres e aquáticos adjacentes. Neste sentido, verificam-se a ocorrência de altos teores de sólidos suspensos e de sulfato em águas fluviais, bem como de metais dissolvidos (em especial ferro (Fe) e Manganês (Mn)), alta acidez e baixos valores de pH (CETEM, 2000; Castilhos et al. 2010; Alexandre, 2000). Castilhos et al. (2010), ao executarem um dos mais recentes monitoramentos das águas e sedimentos superficiais da região carbonífera sul catarinense, indicaram que os metais tóxicos de maior expressão eram o $\mathrm{Fe}$, alumínio $(\mathrm{Al})$, zinco $(\mathrm{Zn})$, cobre $(\mathrm{Cu})$ e mercúrio $(\mathrm{Hg})$.

*Corresponding author: Ricardo Gonçalves Cesar; e-mail: geo_ricardocesar@yahoo.com.br 
Ensaios ecotoxicológicos são comumente empregados na avaliação da qualidade ambiental de ecossistemas aquáticos e terrestres, bem como no estabelecimento de relações entre concentrações de agentes tóxicos no ambiente e efeitos adversos à saúde da flora e da fauna. Uma série de estudos biogeoquímicos e ecotoxicológicos tem sido desenvolvida na região carbonífera sul catarinense (Marcomin, 1996; Alexandre, 2000; CETEM, 2000; Pompêo et al. 2004; Krebs \& Gomes, 2007; Castilhos et al. 2010). Entretanto, a maior parte destes trabalhos privilegiou a avaliação do risco ecológico associado às comunidades aquáticas em detrimento à biota de solo (principalmente a fauna edáfica). Nesse sentido, a execução de bioensaios com organismos terrestres é importante não somente para a elucidação dos riscos à biota do solo, mas sobretudo para complementar os dados já existentes para organismos aquáticos, visando uma avaliação mais ampla do risco ecotoxicológico. Este tipo de informação integrada é fundamental para o planejamento ou tomada de decisão em medidas de saúde pública e de controle ambiental, definição de técnicas de remedição e hierarquização de áreas a serem priorizadas em programas de recuperação ambiental.

No caso dos solos, os oligoquetas (Eisenia andrei - Bouché, 1972) são amplamente empregados em bioensaios, visto que compõem a maior parte da biomassa de fauna de solo, são abundantes em solos tropicais e temperados, ingerem grande quantidade de solo, são de simples cultivo em laboratório e sensíveis à presença de xenobióticos (Hinton \& Veiga, 2002; Liu et al.2005; Nahmani et al. 2007). As hortaliças da espécie Lactuca sativa $\mathrm{L}$. são também frequentemente utilizadas em bioensaios agudos de germinação por necessitarem de um curto período de tempo para seu o brotamento. Os microcrustáceos aquáticos da espécie Daphnia similis (Claus, 1876) são ainda pouco utilizados na avaliação da toxicidade potencial de solos tropicais ou resíduos sólidos, entretanto, são promissores, sobretudo em cenários em que tais materiais possam ser lixiviados e atinjam ecossistemas aquáticos adjacentes, cenário similar à DAM. Para tanto, avalia-se a ecotoxicidade dos lixiviados dos referidos materiais (elutriatos) (Hall et al. 1996, Parkpian et al. 2002, Selivanovskaya \& Latypova 2003, Cesar et al. 2010).

O presente trabalho trata da avaliação ecotoxicológica associada à disposição terrestre de resíduos de mineração de carvão utilizando bioensaios agudos com Eisenia andrei (Bouché, 1972), Lactuca sativa L. e Daphnia similis (Claus, 1876).

\section{MATERIAIS E MÉTODOS}

\section{Amostragem e Preparação dos Materiais}

As amostras de resíduo sólido foram coletadas na bacia hidrográfica do Rio Urussanga (uma das três bacias constituintes da bacia carbonífera sul catarinense). A amostragem foi efetuada em diversas pilhas de rejeito aleatoriamente distribuídas junto à área mais fortemente impactada pela mineração, conforme indicaram Castilhos et al. (2010). Após a remoção da camada superficial (mais intemperizada), a coleta do material foi realizada no interior da pilha. As amostras foram secas à temperatura ambiente e misturadas, de forma a gerar uma única amostra composta. Após essa etapa, a amostra composta foi quarteada e moída em moinho de barras, sendo então enviada para análise química total e ecotoxicológica.

\section{Determinação do pH e dos Teores Totais de Metais}

A determinação do $\mathrm{pH}$ foi realizada através da pesagem de 10 gramas de amostra e posterior adição de $25 \mathrm{~mL}$ de água destilada. Essa mistura foi agitada a 400rpm (agitador orbital) por 30 minutos. Após 20 minutos de repouso, o pH foi determinado no sobrenadante (EMBRAPA, 1997).

A quantificação dos teores totais de zinco $(\mathrm{Zn})$, cobre $(\mathrm{Cu})$, cromo $(\mathrm{Cr})$ e chumbo $(\mathrm{Pb})$ em amostras bióticas e abióticas foi efetuada através da solubilização de 1 grama de amostra seca em uma mistura ácida composta por HF:HCl:HCLO $(2: 1: 1)$ em béquer de platina. A mistura foi então aquecida em chapa a $120^{\circ} \mathrm{C}$, sendo retomada com $\mathrm{HNO}_{3}$ para leitura ICP-OES (HORIBA JOBIN YVON - Ultima 2). A determinação da concentração de mercúrio total $(\mathrm{Hg})$ foi realizada através da pirólise da amostra, utilizando o equipamento LUMEX (RA 915+).

Todas as análises foram realizadas em, pelo menos, duplicata. Amostras certificadas também foram analisadas de modo a garantir a qualidade dos resultados analíticos: NIST 2709 San Joaquin Soil e IAEA 407 - fish homogenate, para amostras abióticas e bióticas, respectivamente. Antes de serem submetidos a tais procedimentos, os oligoquetas foram previamente congelados, liofilizados e triturados.

\section{Avaliação da Intensidade da Poluição por Metais}

A ecotoxicidade do resíduo e a biodisponibilidade de metais foi testada em misturas de resíduo e solos artificiais, o qual é composto por $70 \%$ de areia de quartzo, $20 \%$ de caulim e 10\% de pó de casca de coco (Garcia, 2004). Dessa forma, foram preparadas misturas sólidas do resíduo em solo artificial nas seguintes proporções: $25,30,40,50$ e $70 \%$.

\section{Bioensaio Agudo com Oligoquetas}

O bioensaio agudo com oligoquetas da espécie Eisenia andrei (Bouché, 1972) foi realizado utilizando as misturas solo artificial:resíduo, cujas proporções e preparos encontramse descritos nos itens anteriores. O ensaio foi conduzido com 200 gramas de solo, 10 oligoquetas adultos de peso semelhante (entre 400 e $700 \mathrm{mg}$ - para garantia de populações mais homogêneas) por réplica (3) e umidade do solo ajustada para $50 \%$ da capacidade de campo (ASTM, 2004). Antes de serem introduzidos nos ensaios, os oligoquetas foram deixados sobre papel absorvente umedecido com água destilada, por 24 horas, para o esvaziamento do conteúdo intestinal. Após 14 dias de exposição sob iluminação constante e temperatura controlada $\left(22 \pm 2{ }^{\circ} \mathrm{C}\right)(\mathrm{ASTM}, 2004)$, foi verificada a porcentagem de animais sobreviventes. Os organismos remanescentes foram então enviados para a determinação de metais em seus tecidos. 
A biodisponibilidade potencial de metais foi estudada através do cálculo dos fatores de bioconcentração, definida como a razão entre o teor total do elemento em tecido animal pela concentração no solo.

\section{Ensaio de Germinação com Alface}

As sementes de Lactuca sativa L. utilizadas para este ensaio foram obtidas sem utilização de agrotóxicos. O ensaio foi conduzido com 100 gramas de solo dispostos em pratos plásticos redondos, cuja umidade foi ajustada para a capacidade de campo, com base em OECD (1984). Após a introdução de 40 sementes por réplica (3), 90-100 gramas de areia fina de quartzo foram distribuídas sobre o recipiente-teste, de forma a inibir a perda de umidade e aumentar a agregabilidade do solo.

$\mathrm{O}$ ensaio teve duração de 5 dias, e foi realizado em fotoperíodo de luz e escuro (12h:8h). Os recipientes-teste foram mantidos em sacos plásticos, de modo a minimizar a perda de umidade para a atmosfera. Ao final do ensaio, verificou-se o percentual de sementes germinadas e possíveis alterações morfológicas em sua estrutura.

As doses de resíduo empregadas nestes testes foram baseadas nos resultados obtidos com oligoquetas, visando a utilização das maiores doses capazes de não causar efeitos (mortalidade) aos referidos organismos.

\section{Bioensaio Agudo com Microcrustáceos Aquáticos}

O bioensaio agudo com microcrustáceos da espécie Daphnia similis (Claus, 1876) foi baseado na exposição de fêmeas de seis a 24 horas de idade a lixiviados (elutriatos) do resíduo testado, por um período de 48 horas (ABNT NBR 12713/2004). Para a realização do ensaio, o elutriato foi diluído para as seguintes concentrações: $6,12,24,50$ e $100 \%$. O procedimento adotado para a preparação dos elutriatos está fundamentado nas recomendações de Baun et al. (2002), que propõe a agitação orbital (200 rpm) de uma mistura composta de solo-teste:água (1:8) durante um período de 24 horas. Após essa etapa, a mistura é centrifugada, sendo o sobrenadante filtrado e posteriormente congelado para a avaliação ecotoxicológica.

\section{RESULTADOS E DISCUSSÕES}

\section{Determinação dos Teores de Metais}

A quantificação das concentrações absolutas de $\mathrm{Zn}$ e $\mathrm{Cu}$ no resíduo in natura de mineração de carvão revelou teores abaixo dos obtidos para o solo artificial puro, sendo que os teores de Zn são ordens de grandeza menores do que no solo artificial. As concentrações de $\mathrm{Hg}, \mathrm{Pb}$ e $\mathrm{Cr}$ ficaram acima daquelas encontradas em solo artificial em torno de uma ordem de grandeza, 3 e 5 vezes, respectivamente, e mostram incremento conforme o aumento de proporções do resíduo na mistura. $\mathrm{O}$ valor do $\mathrm{pH}$ seguiu tendência contrária, ou seja, com maiores proporções de resíduo, o valor de $\mathrm{pH}$ decresceu, variando de 3,4 a 4,7 na menor e maior proporções estudadas (25 e 100\%), respectivamente.

A determinação dos teores de metais nas misturas solo artificial:resíduo revelou, para todos os elementos estudados, valores abaixo do limite de prevenção e de intervenção agrícola pela Res. 420 do CONAMA (2009), que estabelece as diretrizes para qualidade de solos no Brasil. Entretanto, alguns tratamentos estão acima do background pedogeoquímico de São Paulo (CETESB, 2005) para Hg $\left(0,050 \mathrm{mg} \mathrm{kg}^{-1}\right)$ e Pb (17 $\mathrm{mg} \mathrm{kg}^{-1}$ ) (Tabela 1).

É importante salientar que o $\mathrm{Hg}, \mathrm{Pb}$ e $\mathrm{Cr}$ são elementos de reconhecida toxicidade, e merecem posição de maior atenção - neste caso, sobretudo o $\mathrm{Hg}$. Por outro lado, as concentrações de metais (em especial o $\mathrm{Zn}$ e o $\mathrm{Cu}$ ) refletem o intenso intemperismo químico e processos de lixiviação aos quais as pilhas de resíduos foram submetidas ao longo do tempo, visto que tais materiais se encontram depositados ao ar livre, estando sujeitos à ação intempérica das águas pluviais associada à geração da DAM. Esta observação sugere que boa parte dos metais tóxicos já foram lixiviados e provavelmente foram acumulados nos solos e/ou sistemas fluviais vizinhos.

\section{Bioensaio agudo com Oligoquetas}

O bioensaio agudo com oligoquetas revelou a ausência de efeitos letais significativos sobre os animais testados (Tabela 2), apesar da diminuição progressiva do $\mathrm{pH}$ com o incremento de proporção de resíduo e do consequente incremento de teores da maior parte dos metais estudados (Tabela 1).

A determinação dos teores $\mathrm{de} \mathrm{Pb}$ e $\mathrm{Cr}$ em tecidos de animais sobreviventes revelou concentrações abaixo do limite de detecção (1,4 e $0,20 \mathrm{mg} \mathrm{kg}^{-1}$ para $\mathrm{Pb}$ e $\mathrm{Cr}$, respectivamente) do método empregado e, por essa razão, não aparecem na Tabela 2. Embora os máximos teores destes metais nos solosteste (i.e., misturas-teste) atinjam 24 e $19 \mathrm{mg} \mathrm{kg}^{-1}$ para $\mathrm{Pb}$ e $\mathrm{Cr}$ (Tabela 1), respectivamente, os teores abaixo dos limites de detecção nos tecidos dos organismos sugerem baixa captação pela biota nas condições testadas.

Tabela 1 - Determinação do $\mathrm{pH}$ e dos teores totais de metais (mg $\mathrm{kg}^{-1}$ ) em resíduo in natura de mineração de carvão, oriundo da bacia

do rio Urussanga (SC), e nas diferentes proporções de mistura solo artificial:resíduo. Notas: \# = solo artificial puro; \#\# = Resíduo moído puro; LR = Limite de referência (background pedogeoquímico do Estado de SP CETESB, 2005); LPA = Limite de prevenção agrícola (CONAMA, 2009); LI = Limite de intervenção (CONAMA, 2009).

\begin{tabular}{lcccccc}
\hline Doses $(\%)$ & $\mathrm{pH}$ & $\mathrm{Hg}$ & $\mathrm{Pb}$ & $\mathrm{Cr}$ & $\mathrm{Cu}$ & $\mathrm{Zn}$ \\
\hline $0^{\#}$ & 5,95 & 0,03 & 13,00 & 7,20 & 6,80 & 48,60 \\
25 & 4,71 & 0,14 & 18,85 & 13,10 & 5,78 & 36,47 \\
30 & 4,63 & 0,16 & 20,02 & 14,28 & 5,57 & 34,04 \\
40 & 4,44 & 0,20 & 22,36 & 16,64 & 5,16 & 29,18 \\
50 & 4,03 & 0,25 & 24,70 & 19,00 & 4,75 & 24,33 \\
$100^{\# \#}$ & 3,40 & 0,46 & 36,40 & 30,80 & 2,70 & 0,060 \\
LR & - & 0,05 & 17 & 40 & 30 & 60 \\
LPA & - & 0,50 & 72 & 75 & 60 & 300 \\
LI & - & 12 & 180 & 150 & 200 & 450 \\
\hline
\end{tabular}


Tabela 2 - Bioensaio agudo com Eisenia andrei em misturas de solo artificial:resíduo de mineração de carvão: níveis de mortalidade dos organismos-teste e determinação das concentrações de metais (em mg $\mathrm{kg}^{-1}$ ) em tecidos de oligoquetas (sobreviventes e antes da exposição). OL = oligoquetas; $\mathrm{FBC}=$ fator de bioconcentração; * = animais diretamente obtidos da cultura.

\begin{tabular}{llllllll}
\hline $\begin{array}{l}\text { Doses } \\
(\%)\end{array}$ & $\begin{array}{l}\text { Mortalidade } \\
(\%)\end{array}$ & $\mathrm{Hg}$ & \multicolumn{3}{l}{$\mathrm{Zn}$} & \multicolumn{3}{c}{$\mathrm{Cu}$} \\
\cline { 2 - 8 } 0 & 0 & $\mathrm{OL}$ & $\mathrm{FBC}$ & $\mathrm{OL}$ & $\mathrm{FBC}$ & $\mathrm{OL}$ & $\mathrm{FBC}$ \\
25 & 0 & 0,01 & 0,33 & 83,25 & 1,71 & 11,25 & 1,65 \\
30 & 2 & 0,05 & 0,34 & 88,60 & 1,23 & 15,20 & 1,71 \\
40 & 3 & 0,04 & 0,25 & 88,90 & 1,36 & 12,70 & 2,05 \\
50 & 0 & 0,06 & 0,29 & 74,50 & 1,08 & 11,40 & 1,20 \\
\multicolumn{2}{l}{$\begin{array}{l}\text { Teores em tecidos animal } \\
\text { antes da exposição* }\end{array}$} & 0,34 & 1,35 & 67,20 & 1,11 & 10,30 & 1,20 \\
\hline
\end{tabular}

Por outro lado, os oligoquetas foram capazes de absorver $\mathrm{Hg}$ do solo, sendo que na maior proporção de resíduo testada com o FBC > 1 (Tabela 1), sugerindo a ocorrência de fenômeno de bioacumulação (Liu et al. 2005). Cesar et al. (2008), Burton et al. (2006) e Cesar et al. (2012), ao executarem bioensaios com E. andrei e E. foetida em solos contaminados por $\mathrm{Hg}$, indicaram que a maior parte dos valores de $\mathrm{FBC}$ para $\mathrm{Hg}$ total são comumente menores do que uma unidade, sendo poucos os autores que reportam valores maiores. $\mathrm{O} \mathrm{Hg}$ não desempenha qualquer função biológica ao metabolismo dos oligoquetas e, desta forma, mesmo em baixas concentrações em tecido, o Hg merece maior atenção no que diz respeito à saúde dos animais expostos.

A determinação dos teores de $\mathrm{Zn}$ e $\mathrm{Cu}$ em tecido animal e a relação com os teores em misturas de rejeito e solo artificial indicou bioacumulação (FBC $>1$ ) (Tabela 2), uma vez que os organismos incorporaram concentrações desses metais acima do existente nas misturas. De fato, as concentrações encontradas em tecidos de animais obtidos junto à cultura (i.e., sem exposição) foram menores se comparados aos animais expostos (Tabela 2). O Zn e o $\mathrm{Cu}$ são metais essenciais ao metabolismo dos oligoquetas e, dessa forma, é válido supor que tais elementos tenham rotas de absorção facilitadas. O Zn desempenha papel vital no crescimento, desenvolvimento e regeneração dos tecidos dos oligoquetas, enquanto o $\mathrm{Cu}$ exerce função no transporte de substâncias entre as células (Lukkari et al. 2005; Suthar \& Singh, 2008). Vale salientar, ainda, que os teores de $\mathrm{Zn}$ e de $\mathrm{Cu}$ nas misturas testadas são oriundos, em sua maior parte, do solo artificial e que a mesma relação dos teores de $\mathrm{Zn}$ e $\mathrm{Cu}$ nos solos são encontrados nos oligoquetas.

As razões dos teores de $\mathrm{Zn}$ e $\mathrm{Cu}$ nas misturas variam em torno de 5 para todas as proporções (Tabela 3). As razões dos teores de $\mathrm{Hg}$ e $\mathrm{Zn}$ bem como as de $\mathrm{Hg}$ e $\mathrm{Cu}$, em todas as proporções, variam de uma a duas ordens, sendo que apenas os solos artificiais mostram três ordens de grandeza para a relação $\mathrm{Zn} / \mathrm{Hg}$. Considerando-se os teores de $\mathrm{Zn}$ e de $\mathrm{Cu}$ nos organismos testados e suas relações, observam-se semelhantes resultados para a relação $\mathrm{Zn} / \mathrm{Cu}$ no solo. A razão $\mathrm{Zn} / \mathrm{Cu}$ para solo:organismo resulta próximo da unidade (Tabela 3). Estas constatações sugerem que o $\mathrm{Zn}$ e o $\mathrm{Cu}$ são absorvidos pelos organismos na mesma proporção que se encontram no solo.
Tabela 3 - Relações (razões) entre as concentrações de zinco (Zn), cobre $(\mathrm{Cu})$ e mercúrio $(\mathrm{Hg})$ encontradas em solos e oligoquetas (Eisenia andrei) sobreviventes do bioensaio agudo.

\begin{tabular}{lccccccccc}
\hline & \multicolumn{1}{c}{$\begin{array}{l}\text { Relação para solos } \\
\text { Doses }\end{array}$} & \multicolumn{4}{c}{$\begin{array}{c}\text { Relação para } \\
\text { organismos }\end{array}$} & \multicolumn{4}{c}{$\begin{array}{c}\text { Relação } \\
\text { solo:organismo }\end{array}$} \\
\cline { 2 - 10 } & $\mathrm{Zn} /$ & $\mathrm{Zn} /$ & $\mathrm{Cu} /$ & $\mathrm{Zn} /$ & $\mathrm{Zn} /$ & $\mathrm{Cu} /$ & $\mathrm{Zn} /$ & $\mathrm{Zn} /$ & $\mathrm{Cu} /$ \\
0 & $\mathrm{Cu}$ & $\mathrm{Hg}$ & $\mathrm{Hg}$ & $\mathrm{Cu}$ & $\mathrm{Hg}$ & $\mathrm{Hg}$ & $\mathrm{Cu}$ & $\mathrm{Hg}$ & $\mathrm{Hg}$ \\
25 & 7 & 1620 & 227 & 7 & 8325 & 1125 & 1 & 0,2 & 0,2 \\
30 & 6 & 261 & 41 & 6 & 1772 & 304 & 1 & 0,1 & 0,1 \\
40 & 6 & 213 & 35 & 7 & 2223 & 318 & 1 & 0,1 & 0,1 \\
50 & 6 & 146 & 26 & 7 & 1242 & 190 & 1 & 0,1 & 0,1 \\
& 5 & 97 & 19 & 7 & 198 & 30 & 1 & 0,5 & 0,6 \\
\hline
\end{tabular}

Por outro lado, quando se comparam as relações $\mathrm{Hg} / \mathrm{Zn}$ e $\mathrm{Hg} / \mathrm{Cu}$ nos solos e também nos organismos, esta relação resulta em 0,1 a 0,2 (Tabela 3), i.e., a absorção de $\mathrm{Hg}$ pelos oligoquetas é uma ordem de grandeza menor do que a absorção de $\mathrm{Cu}$ e $\mathrm{Zn}$, exceto para a relação observada para a maior proporção resíduo:solo testada $(50 \%)$, onde a tendência é de incremento para 0,6 e 0,5 nas relações com o $\mathrm{Zn}$ e $\mathrm{Cu}$, respectivamente (Tabela 3). Na proporção solo:resíduo $50 \%$, há incremento na absorção do Hg pelos oligoquetas, devendo ser melhor investigada. Adicionalmente, deve-se testar maiores proporções possíveis respeitando-se as condições mínimas de alimentação dos oligoquetas.

\section{Teste de Germinação com Alface (Lactuca sativa)}

Os efeitos sobre a germinação foram observados a partir da dose de $50 \%$ (Tabela 4), com perdas de $80 \%$ na germinação observada para a proporção supracitada. Os resultados revelaram, ainda, uma relação positiva entre a diminuição do $\mathrm{pH}$ e a redução do número de sementes germinadas (Tabela 4), sugerindo que o $\mathrm{pH}$ desempenha um papel importante no brotamento das sementes de alface. Além disso, no caso da proporção solo:resíduo $50 \%$, as sementes germinadas apresentaram deformações morfológicas em relação ao controle (plântulas pequenas e pouco desenvolvidas). Adicionalmente, quando o $\mathrm{pH}$ dos solos foi ajustado para a faixa de neutralidade por adição de $\mathrm{CaCO}_{3}$, o número de plântulas (dessa vez, sem deformações) aumentou significativamente, inclusive para a dose de 70\% (Tabela 4).

De modo semelhante a estes resultados, González et al. (2011) também verificaram o aumento do níveis de fitotoxicidade ao executar bioensaios Com $L$. sativa $\mathrm{L}$. em uma área impactada por drenagem ácida de minas, reforçando o papel central da acidez na toxicidade observada. Por outro lado, é importante novamente destacar a influência do $\mathrm{pH}$

Tabela 4 - Teste de germinação com Lactuca sativa utilizando misturas solo:resíduo de mineração de carvão: percentuais de sementes germinadas em função de diferentes doses e respectivos valores de pH (ajustados ou não para a neutralidade).

\begin{tabular}{lcccc}
\hline \multirow{2}{*}{ Dose (\%) } & \multicolumn{2}{c}{$\mathrm{pH}$} & \multicolumn{2}{c}{ Germinação (\%) } \\
\cline { 2 - 5 } 25 & Não Ajustado & Ajustado & Não Ajustado & Ajustado \\
50 & 4,71 & 5,80 & 100 & 100 \\
70 & 4,03 & 6,85 & 22 & 100 \\
\hline
\end{tabular}


sobre a biodisponibilidade potencial de metais; fato que pode também ter contribuído para a redução do número de sementes germinadas.

\section{Bioensaio Agudo com Microcrustáceos Aquáticos}

Os resultados destes ensaios revelaram um incremento de imobilidade dos organismos conforme o decréscimo do $\mathrm{pH}$ dos elutriatos (Tabela 5), sugerindo que este parâmetro desempenhou papel importante também na toxicidade aos microcrutáceos. A redução dos valores de $\mathrm{pH}$ dos elutriatos remete à oxidação da pirita remanescente contida nos resíduos de carvão, e à potencial geração de DAM. Neste sentido, em cenários de lixiviação das pilhas de resíduos via ação intempérica de águas pluviais, estas soluções ácidas mostraram-se capazes de causar dano à biota a partir da concentração acima de 12\% (Tabela 5).

Castilhos et al. (2010), ao realizarem bioensaios com Daphnia magna utilizando águas fluviais coletadas na região carbonífera sul catarinense, também verificaram a existência de uma correlação significativa entre a redução do $\mathrm{pH}$ e o aumento de indivíduos imóveis. Nesse sentido, é importante também salientar que o $\mathrm{pH}$ de fato exerce papel importante no equilíbrio termodinâmico de metais e, consequentemente, na sua biodisponibilidade para microcrustáceos de água doce (Lopes et al., 1999, Rendal et al., 2011) - fato que poderia explicar, ao menos em parte, o aumento da imobilidade proporcional à diminuição do $\mathrm{pH}$. Em ensaios subsequentes, o $\mathrm{pH}$ dos elutriatos deverá ser ajustado para a faixa de neutralidade, de modo a verificar a influência potencial de outros parâmetros, além do $\mathrm{pH}$, na toxicidade. Entretanto, deve-se salientar que o ajuste do $\mathrm{pH}$ pode afetar também a disponibilidade geoquímica dos contaminantes e, consequentemente, seu potencial de assimilação biótica.

\section{CONCLUSÕES E TRABALHOS FUTUROS}

Em um cenário de exposição aguda da pirita (à ação de oxigênio) e dos organismos-teste (às misturas solo:resíduo), foi constatada baixa ecotoxicidade aguda aos oligoquetas edáficos, e baixa disponibilidade de metais altamente tóxicos, como $\mathrm{Cr}$ e o $\mathrm{Pb}$. No caso de $L$. sativa, efeitos significativos sobre a germinação e morfologia foram somente observados em doses muito elevadas $(\sim 50 \%)$ de aplicação no solo.

Tabela 5 - Percentual de fêmeas imóveis de Daphnia similis expostas a diferentes concentrações de lixiviado de resíduo de mineração de carvão, oriundo de pilhas dispostas ao ar livre na bacia do rio Urussanga (SC). ${ }^{\#}=$ Controle do ensaio; ${ }^{\#}=$ Lixiviado in natura .

\begin{tabular}{lccc}
\hline $\begin{array}{l}\text { Concentrações } \\
(\%)\end{array}$ & $\mathrm{pH}$ & $\begin{array}{c}\text { Total de fêmeas } \\
\text { imóveis }\end{array}$ & \% de fêmeas imóveis \\
\hline $0 \#$ & 7,01 & 0 & 0 \\
6 & 6,88 & 0 & 0 \\
12 & 6,41 & 1 & 5 \\
25 & 4,23 & 20 & 100 \\
50 & 3,55 & 20 & 100 \\
$100 \# \#$ & 3,10 & 20 & 100 \\
\hline
\end{tabular}

Por outro lado, é importante ressaltar que estes solos foram testados 24 horas após umedecidos e que, durante esse período de tempo, é possível que somente parte da pirita contida no resíduo tenha sido oxidada, gerando uma DAM menos intensa e de menor toxicidade. Neste sentido, é provável que cenários de exposição prolongada da pirita ao oxigênio (i.e., testar o solo após dias/meses de ser umedecido e aerado) possam resultar no aumento do potencial tóxico desses solos para os oligoguetas.

Tanto para organismos aquáticos como em vegetais, o $\mathrm{pH}$ parece desempenhar, como esperado, papel de suma importância na ecotoxicidade. Em trabalhos futuros, além da caracterização mineralógica do resíduo, deverão ser realizados bioensaios que contemplem uma exposição mais prolongada da pirita ao oxigênio, de modo a estimular a geração mais efetiva de DAM (mais forte) e seu efeito sobre a saúde do ecossistema terrestre.

\section{AGRADECIMENTOS}

Os autores gostariam de agradecer ao Centro de Tecnologia Mineral (CETEM/MCTI) por prover a infra-estrutura laboratorial necessária à execução desta pesquisa. $\mathrm{O}$ apoio financeiro do CNPq, através de concessão de bolsa concedida a Ricardo Cesar (Doutorado/CNPq), Juan Colonese (IC/CNPq), Thiago Alvaro (IC/CNPq) e Mariana Coelho (IC/CNPq), foi igualmente de fundamental importância à realização deste trabalho.

\section{REFERÊNCIAS}

ABNT (Associação Brasileira de Normas Técnicas)- NBR 12713. Ecotoxicologia Aquática - Toxicidade aguda - Método de ensaio com Daphnia spp. (Crustácea, Cladocera), Rio de Janeiro: ABNT 2004.

ALEXANDRE, N.Z., 2000. Análise Integrada da Qualidade das Águias da Bacia do Rio Araranguá (SC). Programa de Pósgraduação em Geografia, Universidade Federal de Santa Catarina, Dissertação de Mestrado.

ASTM (American Society for Testing and Materials)- ASTM E167604. Standard guide for conducting laboratory soil toxicity or Bioaccumulation Tests With The Lumbricid Earthworm Eisenia fetida and the Enchytraeid potworm Enchytraeus albidus, ASTM International 2004.

BAUN, A., JUSTESEN, K.B., NYHOLM, N., 2002. Algal tests with soil suspensions and elutriates: a comparative evaluation for PAH-contaminated soils. Chemosphere 46(2): 251-258. http:// dx.doi.org/10.1016/S0045-6535(01)00097-2

BOUCHÉ, M.B. 1972. Lombriciens de France: Ècologie et systématique. I.N.R.A. Ann. Zool. Ecol. Animal, 72(2): 671. http://dx.doi.org/10.2193/0022541X(2006)70[852:AAMFSE]2.0.CO;2

BURTON, D.T, TURLEY, S.D., FISHER, D.J.U., GREEN, D.J., SHEDD, T.R., 2006, Bioaccumulation of total mercury and monomethylmercury in the earthworm Eisenia fetida. Water Air Soil Pollut., 170: 37-54. http://dx.doi.org/10.1007/s11270-0063113-0

CASTILHOS, Z.C., BIDONE, E.D., CESAR, R.G., EGLER, S.G., BIANCHINNI, M., ALEXANDRE, N.Z., NASCIMENTO, T. 2010. Metodologia de monitoramento da qualidade das águas da Bacia Carbonífera Sul Catarinense: ferramenta para gestão em 
poluição ambiental. In: Série Gestão e Planejamento Ambiental. Rio de Janeiro: CETEM v.1, pp. 03-105

CESAR, R.G., EGLER, S.G., POLIVANOV, H., CASTILHOS, Z.C. , RODRIGUES, A.P., ARAÚJO, P.C., 2008, Biodisponibilidade de metilmercúrio, zinco e cobre em distintas frações granulométricas de solo contaminado utilizando oligoquetas da espécie Eisenia andrei. Anuário do Instituto de Geociências (UFRJ), 31: 33-41.

CESAR, R.G., Alvaro, T.T., SIlva, M.B., COLONESE, J., MOREIRA, C., POLIVANOV, H., EGLER, S.G., BIDONE, E.D., CASTILHOS, Z.C., 2010, Biodisponibilidade de contaminantes em solos brasileiros tratados com lodo de esgoto: uma abordagem ecotoxicológica utilizando bioensaios com organismos aquáticos e edáficos. Geochimica Brasiliensis, 24: 41-49.

CESAR, R., SILVA, M., COLONESE, J., BIDONE, E., EGLER, S., CASTILHOS, Z., POLIVANOV, H., 2012, Influence of the properties of tropical soils in the toxicity and bioavailability of heavy metals in sewage sludge-amended lands. Environ Earth Sci, 66: 2281-2292. http://dx.doi.org/10.1007/s12665-0111449-2

CETEM/CANMET.2000. Projeto Conceitual Preliminar para Recuperação Ambiental da Bacia Carbonífera Sul Catarinense. Relatório Técnico elaborado para o SIECESC (RT33/2000), CETEM/MCTI.

CETESB (Companhia de Tecnologia de Saneamento Ambiental). 2005. In: Valores Orientadores para Solos e Águas Subterrâneas no Estado de São Paulo. Disponível em: <http://www.cetesb. sp.gov.br/Solo/relatorios/tabela_valores_2005.pdf $>$ Accesso em 20 Mar. 2008

CLAUS, C., 1876. Zur Kenntniss der Organisation und des feiner Baues der Daphniiden und verwandten Cladoceren. Zeitschr. Wiss. Zool. 27: 362-402.

CONAMA. 2009. Resolução 420. Disponível em: <http://www. $\mathrm{mma}$. gov.br/port/conama/legiabre.cfm?codlegi $=506>$. Acesso em 19 Out. 2010

EMBRAPA (EMPRESA BRASILEIRA DE PESQUISA AGROPECUÁRIA)., 1997, Centro Nacional de Pesquisa de Solos. In: Manual de Métodos de Análises de Solo. Rio de Janeiro, RJ, pp. 212

GARCIA, M., 2004, Effects of pesticides on soil fauna: Development of ecotoxicology test methods for tropical regions. In: VLEK, P.L.G. et al. (Eds.). Ecology and Development Series, v. 19, Cuvillier Verlag Gottingen, $282 \mathrm{p}$.

GONZÁlEZ, V., GARCÍA, I., DEL MORAL, F., DE HARO, S., SÁNCHEZ, J.A., SIMÓN, M., 2011. Impact of unconfined sulphur-mine waste on a semi-arid environment (Almería, SE Spain). J. Environ Manag., 92(6): 1509-1519. http://dx.doi. org/10.1016/j.jenvman.2011.01.001

HALL, N.E., FAIRCHILD, J.F., POINT, T.W., HEINE, P.H., RUESSLER, D.S.,, INGERSOLL, C.G., 1996, Problems and recommendations in using algal toxicity testing to evaluate contaminated sediments. J. Great Lakes Research 22(3): 545556. http://dx.doi.org/10.1016/S0380-1330(96)70979-6
HINTON, J., VEIGA, M., 2002, Earthworms as bioindicators of mercury pollution from mining and other industrial activities. Geochemistry, Exploration, Environ., Analysis, 2: 269-274. http://dx.doi.org/10.1144/1467-787302-031

KREBS, A.S.J. \& GOMES, C.J.B., 2007, Caracterização Hidroquímica das Águas do Aqüífero Rio Bonito na Região Carbonífera Sul Catarinense. Disponível em: $<$ http://www.cprm. gov.br/rehi/simposio/pa/krebs.pdf>. Acessado em: 18/07/2008.

LIU, X., CHENGXIAO, H., ZHANG, S., 2005, Effects on earthworm activity on fertility and heavy metals bioavailability in sewage sludge. Environ. International, 31: 874-879. http://dx.doi. org/10.1016/j.envint.2005.05.033

LOPES, I., GONÇALVES F., SOARES, A.M.V.M., RIBEIRO, R., 1999. Discriminating the ecotoxicity due to metals and to low ph in acid mine drainage. Ecotoxicol. Environ. Safety, 44:207-214. http://dx.doi.org/10.1006/eesa.1999.1825

LUKKARI, T., ASTSINKI, M., VÄISÄNEN, A., HAIMI, J., 2005, Toxicity of copper and zinc assessed with three different earthworms tests. Appl. Soil Ecol., 30: 133-146. http://dx.doi. org/10.1016/j.apsoil.2005.02.001

MARCOMIN, F.E., 1996, Zoneamento Ambiental do Rio Tubarão SC, através da Análise de Metais Pesados em Água, Sedimento, Substrato, Planta e de Componentes Estruturais da Paisagem. Programa de Pós-graduação em Ecologia, Universidade Federal do Rio Grande do Sul, Dissertação de Mestrado.

NAHMANI, J., HODSON, M.E., BLACK, S., 2007, A review of studies performed to assess metal uptake by earthworms. Environ. Pollut., 145: 402-424. http://dx.doi.org/10.1016/j. envpol.2006.04.009

OECD (Organization for Economic Cooperation and Development), 1984, Guideline 208: Terrestrial Plants, Growth Test. OECD Guidelines for testing of chemical. OECD Paris.

PARKPIAN, O., KLANKRONG, K., DELAUNA, R., JUGSUJÍNDA, A., 2002, Metal leachability from sewage sludge amended Thai soils. J. Environ. Sci. Health, 37: 765-791. http://dx.doi. org/10.1081/ESE-120003588

POMPÊO, M.L.M., MOSCHINI-CARLOS, V., ALEXANDRE, N.Z., SANTO, E., 2004, Qualidade da água em região alterada pela mineração de carvão na microbacia do rio Fiorita (Siderópolis, Estado de Santa Catarina, Brasil). Maringá, 26: 125-136.

RENDAL, C., KUSK, K.O., TRAPP, S., 2011. The effect of pH on the uptake and toxicity of the bivalent weak base chloroquine tested on Salix viminalis and Daphnia magna. Environ. Toxicol. Chemis., 30: 354-359. http://dx.doi.org/10.1002/etc.391

SELIVANOVSKAYA, S.Y., LATYPOVA, V.Z., 2003, The use of bioassays for evaluating the toxicity of sewage sludge and sewage sludge-amended soil. J. Soils Sediments, 3 (2): 85-92. http://dx.doi.org/10.1007/BF02991073

SUTHAR, S., SINGH, S., 2008, Bioconcentrations of metals (Fe, Cu, $\mathrm{Zn}, \mathrm{Pb}$ ) in earthworms (Eisenia fetida), inoculated in Municipal sewage sludge: do earthworms pose a possible risk of terrestrial food chain contamination? Ecotoxicol. Environ. Safety, 24(1): 25-32. http://dx.doi.org/10.1002/tox.20388 